\title{
Invariant decomposition of the retarded electromagnetic field
}

\author{
J. Graells, C. Martín, and J. M. Codina \\ Departament d'Electricitat i Electrònica, Facultat de Física, Universitat de Barcelona, Catalunya, Spain
}

(Received 17 December 1980; accepted for publication 5 April 1985)

\begin{abstract}
The integral representation of the electromagnetic two-form, defined on Minkowski space-time, is studied from a new point of view. The aim of the paper is to obtain an invariant criteria in order to define the radiative field. This criteria generalizes the well-known structureless charge case. We begin with the curvature two-form, because its field equations incorporate the motion of the sources. The gauge theory methods (connection one-forms) are not suited because their field equations do not incorporate the motion of the sources. We obtain an integral solution of the Maxwell equations in the case of a flow of charges in irrotational motion. This solution induces us to propose a new method of solving the problem of the nature of the retarded radiative field. This method is based on a projection tensor operator which, being local, is suited to being implemented on general relativity. We propose the field equations for the pair \{electromagnetic field, projection tensor $\}$. These field equations are an algebraic differential first-order system of oneforms, which verifies automatically the integrability conditions.
\end{abstract}

\section{INTEGRAL REPRESENTATION OF THE ELECTROMAGNETIC FIELD}

It is well known that the vacuum Maxwell equations for the potential one-form $A_{\mu}$ are

$$
A_{\mu, \alpha}{ }^{\alpha}-A_{\alpha, \mu}^{\alpha}=-4 \pi j_{\mu},
$$

from which we can derive the wave equation for the electromagnetic two-form field $F_{\mu \nu}=A_{v, \mu}-A_{\mu, \nu}$,

$$
F_{\mu v, \alpha}{ }^{\alpha}=-4 \pi\left(j_{v, \mu}-j_{\mu, v}\right) \text {. }
$$

We shall make use of this equation because it incorporates the motion of the sources. We must point out that although the law of charge conservation has been lost in (1.2), all our results are consistent with it.

Analyzing the motion of the sources by Fourier transformation, the integral representation of $F_{\mu \nu}$ is obtained in momentum space as

$$
\begin{aligned}
F_{\mu \nu}(x)= & -\frac{4 \pi i}{(2 \pi)^{2}} \int d^{4} k e^{-i k^{\sigma} x_{\sigma}} \\
& \times\left[k_{\mu} j_{v}\left(k^{\sigma}\right)-k_{v} j_{\mu}\left(k^{\sigma}\right)\right] / k^{\sigma} k_{\sigma} .
\end{aligned}
$$

This approach is in the spirit of Huygens and Fresnel. Like them, we consider the field as generated by a distribution of localized sources. The calculus of this integral leads to results that can be found in any advanced textbook, ${ }^{1}$ but transforming back $j_{v}\left(k^{\sigma}\right)$ from momentum space to space-time, Eq. (1.3) becomes

$$
\begin{aligned}
F_{\mu \nu}(x)= & -\frac{4 \pi i}{(2 \pi)^{4}} \int d^{4} \xi \int d^{4} k e^{-i k^{\sigma}(x-\xi)} \\
& \times\left[k_{\mu} j_{v}(\xi)-k_{v} j_{\mu}(\xi)\right] / k^{\sigma} k_{\sigma},
\end{aligned}
$$

which is the retarded field, when suited boundary conditions are imposed. It may seem at a glance that this hybrid expression is not mathematically attractive, but if we look at it from a physical point of view, it can be interpreted as follows: the field generation process at the event $\xi$, and its propagation to the field event $x$, is analyzed in momentum space by means of the density

$$
d^{4} \xi e^{-i k^{\sigma}(x-\xi)_{\alpha}}\left\{\left[k_{\mu} j_{v}(\xi)-k_{v} j_{\mu}(\xi)\right] / k^{\sigma} k_{\sigma}\right\} .
$$

The integration over $k^{\sigma}$ then gives the contribution to the total field generated at $\xi^{\alpha}$, and finally the integration over the domain of definition of the current $j_{\mu}\left(\xi^{\alpha}\right)$ yields to $F_{\mu \nu}(x)$. The possibility of such an interpretation is apparently closely related to the linearity of the theory. This simple interpretation encourages us to go with our purpose to study geometrically the retarded radiative field and to generalize the expression of the electromagnetic field created by a structureless point charge in a given arbitrary motion.

Our starting point will be the integral on momentum space. With respect to a global inertial frame the volume element splits into $d^{4} k=d^{3} \mathbf{k} d k^{0}$. Now, we can analytically continue $k^{0}$ to the complex plane, and applying the residue theorem, by choosing the retarded prescription, it is obtained:

$$
\begin{aligned}
F_{\mu \nu}(x)= & \frac{1}{(2 \pi)^{2}} \int d^{4} \xi \theta\left(x^{0}-\xi^{0}\right) \int \frac{d^{3} \mathbf{k}}{|\mathbf{k}|}\left\{e^{-k^{k^{\sigma}}(x-\xi)_{\sigma}}\right. \\
& \left.\times \underset{+}{\times(k \wedge j(\xi))_{\mu \nu}+e^{-k^{k^{\sigma}}}(x-\xi)_{\sigma}}(k \wedge j(\xi))_{\mu \nu}\right\},
\end{aligned}
$$

where we have defined the following.

(1) ${ }^{k^{\sigma}}+\equiv(|\mathbf{k}|, \mathbf{k}), \stackrel{k^{\sigma}}{-} \equiv(-|\mathbf{k}|, \mathbf{k})$ are the lightlike vectors. duct.

(2) $(k \wedge j(\xi))_{\mu \nu} \equiv k_{\mu} j_{\nu}(\xi)-k_{v} j_{\mu}(\xi)$ is the exterior pro-

(3) $\theta\left(x^{0}-\xi^{0}\right)$ is the Heaviside or step function. It is understood that the Minkowski metric has signature +2 , i.e.,

$$
k^{\sigma} k_{\sigma}=\eta_{\alpha \beta} k^{\alpha} k^{\beta}=-\left(k^{0}\right)^{2}+\mathbf{k}^{2} .
$$

Until now, this approach is equivalent to the propagator (Green's function) approach.

Taking into account that $d w=d^{3} \mathbf{k} /|\mathbf{k}|$ is the measure of the upper light cone on momentum space, Eq. (1.5) can be written geometrically

$$
\begin{aligned}
F(x)= & \frac{1}{(2 \pi)^{2}} \int d^{4} \xi \theta\left(x^{0}-\xi^{0}\right) \int_{k^{\sigma} k_{\sigma}=0} d w \epsilon\left(k^{0}\right) \\
& \times e^{i k^{\sigma}(x-\xi)_{\sigma}} k \wedge j(\xi),
\end{aligned}
$$

where $\epsilon\left(k^{0}\right)$ is the signum function. 
It is clear in Eq. (1.6) that the integral over the momentum light cone is the exterior differential of the invariant Jordan-Pauli distribution, ${ }^{2}$ therefore we can reduce the integral to the upper light cone $k^{\sigma} k_{\sigma} \theta\left(k^{0}\right)=0$, by virtue of $\theta\left(x^{0}-\xi^{0}\right)$. In order to calculate it, we shall make use of the following orthonormal tetrad field: $\left\{e_{i}\right\}=\left\{e_{0}, e_{1}, e_{2}, e_{3}\right\}$, which is defined at the generic source event $\xi$ according to the following.

(a) $e_{0} \equiv v$ is the current four-vector velocity, i.e., if $\rho_{0}$ is the proper charge density, then $j^{\mu}=\rho_{0} v^{\mu}, v^{\mu} v_{\mu}=-1$.

(b) $e_{1}$ is a unit spacelike vector $e_{1}^{\alpha} e_{1 \alpha}=1$, orthogonal to $e_{0}$ and included in the plane determined by $e_{0}$ and the separation vector $R^{\alpha}=(x-\xi)^{\alpha}$.

(c) $e_{2}$ and $e_{3}$ are two unit orthogonal spacelike vectors $e_{2}^{\alpha} e_{2 \alpha}=e_{3}^{\alpha} e_{3 \alpha}=1, e_{2}^{\alpha} e_{3 \alpha}=0$, the plane they determine being perpendicular to that defined by $\left\{e_{0}, e_{1}\right\}$.

The dual basis $\left\{\omega^{i}\right\}$ to $\left\{e_{i}\right\}$ is canonically defined by

$$
\left\langle\omega^{i}, e_{j}\right\rangle=\delta_{j}^{i},
$$

where $\delta_{j}^{i}$ is the Kronecker delta. Care must be taken because the velocity $v$, considered as a one-form, is related to $\omega^{0}$ with a minus sign: $\omega_{\alpha}^{0}=-v_{\alpha}$.

The separation vector $R=(x-\xi)$ between the field event $x$ and the source one $\xi$ is expressed with respect to the tetrad $\left\{e_{i}\right\}$ by

$$
R \equiv x-\xi=r e_{0}+r^{\prime} e_{1},
$$

where $r=-e_{0} \cdot R$ and $r^{\prime}=e_{1} \cdot R$ are the Lorentz-invariant tetrad components of $R$.

In order to compute the integral over the upper light cone

$$
I(\xi) \equiv \int_{k^{\sigma} k_{o} \theta\left(k^{0}\right)=0} d w e^{-i k^{\sigma}(x-\xi)_{\sigma}} k \wedge j(\xi),
$$

we make use of standard calculations. For example, by choosing spherical coordinates at the simultaneity $\xi^{0}=c^{\Gamma_{e}}$, with polar axis $e_{1}$, it is easily obtained

$$
\begin{aligned}
I(\xi)= & 4 \pi i \int_{0}^{\infty} d|\mathbf{k}| e^{i|\mathbf{k}| r} \\
& \times\left(|\mathbf{k}| \frac{\cos |\mathbf{k}| r^{\prime}}{r^{\prime}}-\frac{\sin |\mathbf{k}| r^{\prime}}{r^{\prime}}\right) e_{1} \wedge j(\xi) .
\end{aligned}
$$

Now, according to the Laplace-Carlson transform, ${ }^{3}$

$$
\begin{aligned}
& p \int_{0}^{\infty} d t e^{-p^{t}} \sin a t=\frac{a p}{p^{2}+a^{2}}, \\
& p \int_{0}^{\infty} d t e^{-p^{t}} t^{v-1} \cos a t \\
& \quad=\frac{\Gamma(v)}{2} p\left(\frac{1}{(p-i a)^{v}}+\frac{1}{(p+i a)^{v}}\right) .
\end{aligned}
$$

Insertion of $p=-i r, a=r^{\prime}, v=2, \Gamma(2)=1, t=|\mathbf{k}|$ into the above integrals yields

$$
\begin{aligned}
I(\xi)= & -4 \pi i \frac{1}{r^{\prime}}\left(\frac{r^{2}+r^{2}}{\left(-r^{2}+r^{\prime 2}\right)^{2}}+\frac{1}{-r^{2}+r^{\prime 2}}\right) \\
& \times e_{1}(\xi) \wedge j(\xi) .
\end{aligned}
$$

Inserting the expression (1.9) into the electromagnetic field (1.6), we get the equation

$$
\begin{aligned}
F(x)= & -\frac{4 \pi i}{(2 \pi)^{2}} \int d^{4} \xi \theta\left(x^{0}-\xi^{0}\right) \frac{1}{r^{\prime}} \\
& \times\left(\frac{r^{2}+r^{\prime 2}}{\left(-r^{2}+r^{2}\right)^{2}}+\frac{1}{\left(-r^{2}+r^{\prime 2}\right)}\right) e_{1}(\xi) \wedge j(\xi) .
\end{aligned}
$$

\section{LOCALLY PROPER TIME SYNCHRONIZABLE FLOW OF CHARGES}

At a glance it may seem that in order to derive consequences of expression (1.10), it is necessary to know $j(\xi)$, but on second thought if we dare try some general properties of the flow of charges that constitute the current density, an interesting expression of the electromagnetic two-form can be derived.

We first assume for a current density $j=\rho_{0} v$, that its velocity one-form field $v$ verifies Frobenius condition ${ }^{4}$

$$
v \wedge d v=0 .
$$

From a physical point of view it means that the observers associated to the $v$ timelike flow make a locally synchronizable frame. This condition result is too general, therefore, we furthermore assume the more restrictive one

$$
d v=0,
$$

which means that the flow is locally proper time synchronizable, i.e., the observers reference frame can experimentally correlate by "radar" their proper times. In other words, there exists a family of three-spaces to which the streamlines are orthogonal. This motion is an irrotational one, i.e., in decomposing that portion of the covariant derivative $v_{\alpha ; \beta}$ which is perpendicular to the velocity, into its antisymmetric part, its symmetric trace-free part, and the trace itself ${ }^{5}$

$$
v_{\alpha ; \beta}=-v_{\alpha ; \gamma} v^{\gamma} v_{\beta}+\omega_{\alpha \beta}+\sigma_{\alpha \beta}+\frac{1}{3} \vartheta h_{\alpha \beta} .
$$

The rotation reduces to $\omega=a \wedge v$. Now, according to the global version of the Frobenius theorem, due to Chevalley and Ehresman, applied to the present case, it implies that Minkowski space-time is foliated by the integral manifolds (three-spaces above) of the vector distribution defined by the velocity field $v: x \rightarrow M_{x}$. This foliation can be labeled by the proper time $\tau$ coordinate $(v=d \tau)$ and three spacelike coordinates $\boldsymbol{\eta}^{i}$ suited to the three-spaces.

Therefore, we refer Minkowski space-time to the inertial coordinate $\operatorname{system}\left(\xi^{0}, \xi^{i}\right)$ and to that induced by the foliation $\left(\tau, \eta^{i}\right)$

$$
\left\{\begin{array} { l } 
{ \xi ^ { 0 } = \xi ^ { 0 } ( \tau , \eta ^ { i } ) , } \\
{ \xi ^ { i } = \xi ^ { i } ( \tau , \eta ^ { i } ) , }
\end{array} \quad \left\{\begin{array}{l}
\tau=\tau\left(\xi^{0}, \xi^{i}\right) \\
\eta_{i}=\eta^{i}\left(\xi^{0}, \xi^{i}\right) .
\end{array}\right.\right.
$$

We can bring the Minkowski line element with respect to the coordinates $\left(\tau, \eta^{i}\right)$ to the form

$$
d s^{2}=-d \tau^{2}+g_{i j}\left(\tau, \eta^{i}\right) d \eta^{i} d \eta^{i}=-\left(d \xi^{0}\right)^{2}+(d \xi)^{2},
$$

because $v^{\alpha}$ is not a null vector. The volume element will be expessed as

$$
d^{4} \xi=\sqrt{|g|} d \tau d^{3} \eta=\sqrt{g} d \tau d^{3} \eta .
$$

With these premises, Eq. (1.10) can be split into 


$$
\begin{aligned}
F(x)= & \frac{1}{\pi i} \int d^{3} \eta \int d \tau \sqrt{g} \theta\left(x^{0}-\xi^{0}\right) \\
& \times \frac{1}{e_{1} \cdot R}\left(\frac{(v \cdot R)^{2}+\left(e_{1} \cdot R\right)^{2}}{\left(R_{\alpha} R^{\alpha}\right)^{2}}+\frac{1}{R_{\alpha} R^{\alpha}}\right) e_{1} \wedge j .
\end{aligned}
$$

The integration over $\tau$ will be done by applying the residue theorem. The Heaviside function selects the retarded pole, but the difference from the previous calculation (integral over $\left.k^{0}\right)$ consists in the fact that the denominator $\left(R^{\alpha} R_{a}\right)^{2}$ now vanishes to the second order (pole of order 2 ), so that we have to carry the expansion to the order $\left(\tau-\tau_{\mathrm{r}}\right)^{2}$ (where " $\mathrm{r}$ " stands for "retarded")

$$
\begin{aligned}
R_{\alpha} R^{\alpha}= & -\left(R_{\alpha} v^{\alpha}\right)_{\tau_{\mathrm{r}}}\left(\tau-\tau_{\mathrm{r}}\right)-\left(1+R_{\alpha} a^{\alpha}\right)_{\tau_{\mathrm{r}}} \\
& \times\left(\tau-\tau_{\mathrm{r}}\right)^{2}+O\left[\left(\tau-\tau_{\mathrm{r}}\right)^{3}\right] .
\end{aligned}
$$

Inserting the development (2.7) into the expression (2.6), it reduces to

$$
\begin{aligned}
F(x)= & \frac{1}{\pi i} \int d^{3} \eta(-2 \pi i) \lim _{\tau \rightarrow \tau_{\mathrm{r}}}\left\{\left(\tau-\tau_{r}\right) \frac{\sqrt{g}}{\epsilon_{1} \cdot R} \frac{e_{1} \wedge j}{-2 R_{\alpha} v^{\alpha}\left(\tau-\tau_{\mathrm{r}}\right)+O\left[\left(\tau-\tau_{\mathrm{r}}\right)^{2}\right]}\right. \\
& \left.+\frac{\partial}{\partial \tau}\left(\left(\tau-\tau_{\mathrm{r}}\right)^{2} \cdot \frac{\sqrt{g}}{e_{1} \cdot R} \frac{(v \cdot R)^{2}+\left(e_{1} \cdot R\right)^{2}}{-2 R_{\alpha} v^{\alpha}\left(\tau-\tau_{\mathrm{r}}\right)-\left(1+R_{\alpha} a^{\alpha}\right)\left(\tau-\tau_{\mathrm{r}}\right)^{2}+O\left[\left(\tau-\tau_{\mathrm{r}}\right)^{2}\right]} e_{1} \wedge j\right)\right\} .
\end{aligned}
$$

This method of calculus follows the Sommerfeld approach to the residue theorem. ${ }^{6}$ We now introduce the lightlike vector $L=e_{1}+v$, which verifies the following algebraic relations:

$$
L \cdot L=0, \quad L \cdot e_{1}=1, \quad L \cdot v=-1 .
$$

Differentiating these relations along the flow worldline, i.e., with respect to $\tau$, and making simple algebraic operations, we obtain

$$
\frac{\partial e_{1}^{\alpha}}{\partial \tau} \equiv\left(\nabla_{v} e_{1}\right)^{\alpha}=-a^{\alpha}+\left(a \cdot e_{1}\right) L^{\alpha} .
$$

Taking into account the law of charge conservation $\partial_{\alpha} J^{\alpha}=0$, we can easily derive

$$
\frac{\partial \rho_{0}}{\partial \tau}=\nabla_{v} \rho_{0}=-\rho_{0} \nabla \cdot v
$$

and, finally, applying the equality

$$
\frac{1}{g} \frac{\partial g}{\partial \tau}=\nabla \cdot v
$$

it allows us to express Eq. (2.8) as we desire

$$
\begin{aligned}
F(x)= & -\int d^{3} \eta\left(\sqrt{g} \rho_{0} \frac{e_{1} \wedge L}{(-v \cdot R)^{2}}\right)_{\tau_{\mathrm{r}}} \\
& +\frac{1}{2} \int d^{3} \eta\left(\sqrt{g} \nabla \cdot v \rho_{0} \frac{e_{1} \wedge L}{(-v \cdot R)}\right)_{\tau_{\mathrm{r}}} \\
& +\int d^{3} \eta\left(\sqrt{g} \rho_{0} \frac{a+\left(a \cdot e_{1}\right) v}{(-v \cdot R)} \wedge L\right)_{\tau_{\mathrm{r}}} .
\end{aligned}
$$

Until now we have not dealt with the question of the integral domain of the $\eta^{i}$ coordinates. The calculus of the residue has implied that this domain is defined by the equations

$$
\left\{\begin{array} { l } 
{ \tau _ { \mathrm { r } } = \tau ( x ^ { 0 } - | \mathbf { x } - \xi | , \xi ^ { j } ) , } \\
{ \eta ^ { i } = \eta ^ { i } ( x ^ { 0 } - | \mathbf { x } - \xi | , \xi ^ { j } ) , }
\end{array} \Leftrightarrow \left\{\begin{array}{l}
\xi^{0}=x^{0}-|\mathbf{x}-\xi|, \\
\xi^{j}=\xi^{j} .
\end{array}\right.\right.
$$

Therefore it is clearly seen that the integration domain is the retarded light cone of the field event: $I_{r}(x)$.

Now, following Newman and Penrose, ${ }^{7}$ a complex null tetrad $\{z\}=\{l, n, m, \bar{m}\}$ is introduced for expressing Eq. (2.12), because of its adequacy and internal adaptability for studying the solution and the equations of massless fields possessing certain algebraic properties.

The Newman-Penrose tetrad is defined by the standard prescription

$$
\begin{aligned}
& l=(1 / \sqrt{2})\left(v+e_{1}\right) \equiv L / \sqrt{2}, \quad v=(1 / \sqrt{2})(l+n), \\
& n=(1 / \sqrt{2})\left(v-e_{1}\right), \quad e_{1}=(1 / \sqrt{2})(l-n), \\
& m=(1 / \sqrt{2})\left(e_{2}+i e_{3}\right), \quad e_{2}=(1 / i \sqrt{2})(m-\bar{m}), \\
& \bar{m}=(1 / \sqrt{2})\left(e_{2}-i e_{3}\right), \quad e_{3}=(1 / \sqrt{2})(m+\bar{m}) .
\end{aligned}
$$

With respect to this tetrad the acceleration $a$ is expressed as

$$
\begin{aligned}
a & =a^{1} e_{1}+a^{2} e_{2}+a^{3} e_{3} \\
& =(1 / \sqrt{2})\left(a^{1} l-a^{1} n+\bar{A} m+A \bar{m}\right),
\end{aligned}
$$

where we have defined $A=\left(a^{3}+i a^{2}\right) ; \bar{A}$ is the complex conjugate of $A$.

Therefore, Eq. (2.12) will reduce to

$$
\begin{aligned}
F(x)= & \int_{I_{r}(x)} d^{3} \eta \sqrt{g} \rho_{0} \frac{n \wedge l}{r^{2}} \\
& +\frac{1}{2} \int_{I_{r}(x)} d^{3} \eta \sqrt{g} \rho_{0} \nabla \cdot v \frac{n \wedge l}{r} \\
& +\int_{I_{r}(x)} d^{3} \eta \sqrt{g} \rho_{0} \frac{(\bar{A} m+A \bar{m})}{r} \wedge l .
\end{aligned}
$$

At this stage we must point out that the Newman-Penrose (NP) tetrad is associated with the flow of charges, but in the one-point structureless charge case is directly associated to $F$. In fact, for this particular and well-studied problem, the integration is made trivially because it reduces to the intersection of the $q$ charge worldline with the retarded light cone $I_{r}(x)$, and taking into account that $\nabla \cdot v=0$, Eq. (2.16) is in this case

$$
F_{q}(x)=q\left(n \wedge l / r^{2}\right)_{\tau_{\mathrm{r}}}+q((\bar{A} m+A \bar{m}) \wedge l / r)_{\tau_{\mathrm{r}}} .
$$

We remember here that every magnitude refers to the retarded event $z^{\alpha}\left(\xi_{r}^{0}\right)=z^{\alpha}\left(x^{0}-|x-\xi|\right)$ of the $x$ field event, $z^{\alpha}\left(\xi^{0}\right)$ being the trajectory of the $q$-point charge. Now the Newman-Penrose tetrad is associated with the light congruence of $F$, whose tangent null vector field is $l$. 
The matrix representation of Eq. (2.17) with respect to the n.p. tetrad is

$$
\begin{aligned}
(F)= & \frac{q}{r^{2}}\left(\begin{array}{r:rr}
0 & -1 & 0 \\
1 & 0 & 0 \\
\hdashline 0 & 0
\end{array}\right) \\
& +\frac{q}{r}\left(\begin{array}{r:rr}
0 & -\bar{A} & A \\
\hdashline \bar{A} & 0 & 0 \\
-A & 0 & 0
\end{array}\right) .
\end{aligned}
$$

As was shown by Newman and Penrose, this approach is equivalent to the spinor formalism. The consequence for the one-point charge have been widely studied in the literature. For this reason, we now turn back to the much more general and not-studied case, represented by Eq. (2.16). We must remember that the integrals extend to the lower light cone of the field event, parametrized by a generic spacelike slice of the foliation induced by $v$. The splitting of the two-form $F=F_{0 i} d t \wedge d x^{i}+F_{i j} d x^{i} \wedge d x^{j}$, which lets us identify $\left(F_{0 i}\right)=\mathbf{E}$ as the electric field and $\left(F_{i j} e^{i j k}\right)=\mathbf{B}$ as the magnetic field, only has meaning with respect to an inertial frame! Therefore, we must refer Eq. (2.16) to an inertial frame. Then, writing down the vector components by Greek letters, we obtain, returning back to source kinematical variables

$$
\begin{aligned}
F_{\alpha \beta}(x)= & \int_{I_{\mathrm{r}}(x)} d w \rho_{0} \frac{v_{\alpha} e_{1} \beta-v_{\beta} e_{{ }_{1} \alpha}}{r}+\frac{1}{2} \int_{I_{\mathrm{r}}(x)} d w \rho_{0} \\
& \times \nabla \cdot v\left(v_{\alpha} e_{{ }_{1} \beta}-v_{\beta} e_{1 \alpha}\right)+\int_{I_{r}(x)} d w \rho_{0}\left[\left(a_{\alpha} v_{\beta}-a_{\beta} v_{\alpha}\right)\right. \\
& \left.-\left(e_{1 \alpha}\left(a^{1} v_{\beta}+a_{\alpha}\right)-e_{1 \beta}\left(a^{1} v_{\alpha}+a_{2}\right)\right)\right],
\end{aligned}
$$

where we have taken into account that $d w=\sqrt{g}\left(d^{3} \eta /-v \cdot R\right)$ is the measure or absolute Lorentz-invariant two-content of the retarded null cone of the field event.

The meaning of the three-integral splitting of Eq. (2.19) is as follows:

generalized coulomb field,

$$
C_{\alpha \beta} \equiv \int_{I_{\Gamma}(x)} d w \rho^{0} \frac{v_{\alpha} e_{1 \beta}-v_{\beta} e_{1 \alpha}}{r} ;
$$

intermediate-longitudinal field,

$$
I_{\alpha \beta} \equiv \int_{I_{r}(x)} d \omega \frac{1}{2} \rho_{0} \nabla \cdot v\left(v_{\alpha} e_{1 \beta}-v_{\beta} e_{1 \alpha}\right)
$$

radiation field,

$$
\begin{aligned}
R_{\alpha \beta} \equiv & \int_{I_{r}(x)} d \omega \rho_{0}\left[\left(a_{\alpha} v_{\beta}-a_{\beta} v_{\alpha}\right)-\left(e_{1 \alpha}\left(a^{1} v_{\beta}+a_{\beta}\right)\right.\right. \\
& \left.\left.-e_{1 \beta}\left(a^{1} v_{\alpha}+a_{\alpha}\right)\right)\right] .
\end{aligned}
$$

The justification of $C_{\alpha \beta}, I_{\alpha \beta}, R_{\alpha \beta}$ becomes apparent when one goes to the rest system of the integrand two-forms. In this system, it is verified at the fixed source event we work on: $v_{\alpha}=(-1,0), e_{1}=(0, \mathbf{r} /|\mathbf{r}|) ; \mathbf{r}$ being the three-vector which points from the retarded position of the flow of charges to the field point. The Coulomb and radiation fields are direct generalizations of the one-point structureless charge.

The intermediate-longitudinal field is a new one, which has a structure, being longitudinal, similar to the Coulomb field, but it depends on $r$, like a radiation field. Then the Lorentz-invariant separation between velocity and acceleration-radiation fields is only possible for the one-point charge case.

\section{A NEW APPROACH: PROJECTION OPERATOR}

The electromagnetic radiation field appears in a number of different forms: acceleration-retarded fields (kinematical source criteria); free fields (dynamical criteria), e.g., $F$ $=F_{\text {retarded }}-F_{\text {advanced }}$; null fields (algebraic criteria); asymptotic developments (Goldberg-Keer theorem, B.M.S. group); etc. None of these methods scarcely may be completely implemented in general relativity, and they are not always equivalent among themselves. Then we consider it quite reasonable to ask ourselves: What do we mean by "radiative field"? Perhaps this question is a methodological one, but even in this case, we must remember what Ginzburg ${ }^{8}$ said: "In Physics there are many 'perpetual problems' the discussion of which continues for decades... . On the other hand, however, neglect of such methodological types of problems sometimes incurs vengeance!' Our approach to dealing with the problem, i.e., to defining what the radiative field is for us, is a dynamical geometrical one, which takes into account that when the electromagnetic field produced by a given source is measured, one always finds the retarded field, and it shares the maximum number of common features of all the above criteria.

For the moment we shall not invoke "Occam's razor," and shall assume the existence of a projection tensor operator $P^{\alpha \beta}{ }_{\gamma \delta}$, such that when applied to the total electromagnetic field gives us the desired radiative part

$$
R_{\alpha \beta} \equiv P_{\alpha \beta}{ }^{\gamma \delta} F_{\gamma \delta} .
$$

It is easily found that $P_{\alpha \beta \gamma \delta}$ is antisymmetric in each of the index pairs $\alpha \beta$ and $\gamma \delta$, but is symmetric under interchange of the pairs.

It is clear that the problem now has been shifted to find $\boldsymbol{P}_{\alpha \beta \gamma \delta}$. What are the advantages to introducing this new entity? First of all, if we can find a reasonable system of firstorder partial differential equations for it, we shall have a criteria based on dynamical-geometric equations that when applying the strong equivalence principle will be valid in general relativity. Second, it is therefore a local field criteria. Third, it implies a new concept of the electromagnetic field, which we view now as the pair $\{P, F\}$, the contraction $\langle P, F\rangle$ being the radiative part.

The algebraic-partial differential system that we propose is as follows:

$$
\begin{aligned}
& * F_{[\alpha \beta, \gamma]}-4 \pi * j_{[\alpha \beta \gamma]}=0, \\
& F_{[\alpha \beta, \gamma]}=0, \\
& P_{\alpha \beta \mu \nu}-P_{\alpha \beta \gamma \delta} P^{\gamma \delta}{ }_{\mu v}=0, \\
& P_{\alpha \beta \mu \nu} F^{\alpha \beta} F^{\mu \nu}=0, \\
& \epsilon_{\alpha \beta \gamma \delta} P^{\alpha \beta \mu \nu} P^{\gamma \delta \tau \sigma} F_{\mu \nu} F_{\tau \sigma}=0, \\
& P_{[\alpha \beta}{ }^{\gamma \delta} F_{\gamma \delta, v]}+P_{[\alpha \beta, \nu]}^{\gamma \delta} F_{\gamma \delta}=0,
\end{aligned}
$$

and boundary conditions in the Sommerfeld sense. We have to mention that, because there is some confusion in the literature. Sommerfeld said quite explicitly that his aim is to ex- 
clude incoming radiation. Retarded solutions for spatially bounded sources satisfy his conditions automatically in future null directions. $\left(\mathscr{I}^{+}\right.$is the "future null infinity," the region $t+|\mathbf{r}| \rightarrow+\infty$ at finite $t-|\mathbf{r}|$.)

In system (3.2), the first two equations are Maxwell equations. The third equation simply states that $P$ is a projection tensor. The fourth and fifth equations mean that the radiative field has to be degenerate or algebraically special (they are equivalent to $F_{\alpha \beta} F^{\alpha \beta}=0, * F_{\alpha \beta} F^{\alpha \beta}=0$ ). The sixth equation states that the radiative field is closed. As far as the boundary conditions are concerned, we adopt the Sommerfeld point of view, and explicitly we exclude the homogeneous-free solutions of the system. Then as Fock has remarked, care must be taken at past infinity, because as one recedes along null straight lines coming in from the past, the retarded field reflects source behavior at even earlier times; a condition on the time dependence of the sources in the past infinity is required in order that the retarded field satisfies Sommerfeld's condition at past null infinity. ${ }^{9}$

We have studied the system (3.2) from the point of view of the differential ideal of Frobenius-Cartan theory, i.e., as an exterior differential system. After a lengthy but easy calculation we have proved that the system verifies the required integrability conditions, and consequently has solutions in any given Riemannian spacetime, i.e., in the presence of gravitational fields. (In the Appendix to the present article we sketch the proof.)

For the one-point structureless charge problem, we have found the solution. The projection tensor referring to the dual bases of the orthonormal tetrad defined in Sec. I is

$$
P=-\left(\omega^{0} \wedge \omega^{2}\right)\left(\omega^{0} \wedge \omega^{2}\right)+\left(\omega^{1} \wedge \omega^{2}\right)\left(\omega^{1} \wedge \omega^{2}\right),
$$

being expressed with respect to the global inertial frame as follows:

$$
\begin{aligned}
P_{\alpha \beta \mu \nu}= & {\left[1 /\left(a^{2}\right)^{2}\right] \delta_{\alpha \beta}^{\alpha^{\prime} \beta^{\prime}} \delta_{\mu v}^{\mu^{\prime} v^{\prime}}\left\{-\left(v_{\alpha^{\prime}} a_{\beta^{\prime}}-a^{1} v_{\alpha^{\prime}} e_{1 \beta^{\prime}}\right)\right.} \\
& \left.\times\left(v_{\mu^{\prime}} a_{v^{\prime}}-a^{1} v_{\mu^{\prime}} e_{1 \alpha^{\prime}}\right)+\left(e_{1 \alpha^{\prime}} a_{\beta^{\prime}} e_{1 \mu^{\prime}} a_{v^{\prime}}\right)\right\}
\end{aligned}
$$

where $\delta_{\alpha \beta}^{\alpha^{\prime} \beta^{\prime}}=\delta_{\alpha}^{\alpha^{\prime}} \delta_{\beta}^{\beta^{\prime}}-\delta_{\beta}^{\alpha^{\prime}} \delta_{\alpha}^{\beta^{\prime}}$, and the orthonormal basis has been chosen so that $a=a^{1} e_{1}+a^{2} e_{2}$. Functionally $P$ must be considered as depending on the field event $x$ through the retarded prescription, i.e., $P(x)=P\left(\tau_{\mathrm{r}}(x)\right)$.

The physical and mathematical structure of the projection tensor which corresponds to the electromagnetic field derived in Sec. II of this article can be easily determined if we is taken into account its similarity with the one-point charge solution; in fact, the Coulomb and intermediate two-form integrands are always bivector orthogonal to the radiation two-form integrand.

According to the existence theorem for the system (3.2), it shall be possible to find a tetrad field of one-forms $\left\{W^{i}(x)\right\}$ to refer to the cotangent bundle associated to Minkowski space-time. With respect to this tetrad field, Eq. (2.19) may be expressed as

$$
\begin{aligned}
F(x)= & (C I)(x)\left\{W^{0}(x) \wedge W^{1}(x)\right\} \\
& +R(x)\left\{\left(W^{0}(x)-W^{1}(x)\right) \wedge W^{2}(x)\right\},
\end{aligned}
$$

where

$(C I)(x) W^{0} \wedge W^{1} \equiv-\int_{I_{r}(x)} d \omega \rho_{0}\left[\frac{1}{r}+\frac{1}{2} \nabla \cdot v\right] \omega^{0} \wedge \omega^{1}$,

$$
R(x)\left(W^{0}-W^{1}\right) \wedge W^{2} \equiv \int_{I_{r}(x)} d \omega \rho_{0}\left(\omega^{0}-\omega^{1}\right) \wedge \omega^{2} .
$$

Therefore the projection tensor operator will be

$P(x)=-\left(W^{0} \wedge W^{2}\right)\left(W^{0} \wedge W^{2}\right)+\left(W^{1} \wedge W^{2}\right)\left(W^{1} \wedge W^{2}\right)$.

It is interesting to remark that the radiative solution implied by Eqs. (3.6) and (3.7) obeys at least the same properties of usual null fields. In fact, with respect to a global inertial frame $\left\{x^{\alpha}\right\}$, where it is defined $W^{0}(x)-W^{1}(x) \equiv l_{\alpha} d x^{\alpha}$, the radiative field will be expressed as

$$
R_{\alpha \beta}=R(x) l_{\alpha} \wedge W_{\beta}^{2}(x)
$$

Evidently, here $l_{\alpha}$ is a null one-form: $l^{\alpha} l_{\alpha}=0$, which belongs to the congruence associated to $R_{\alpha \beta}(x)$. Its impulseenergy tensor reduces to

$$
\begin{aligned}
T_{\mu \nu}^{(R)} & =(1 / 4 \pi)\left(R_{\mu \lambda} R_{\nu}^{\lambda}-\frac{1}{4} \eta_{\mu \nu} R_{\alpha \beta} R^{\alpha \beta}\right) \\
& =\frac{R^{2}(x)}{4 \pi} l_{\mu}(x) l_{v}(x),
\end{aligned}
$$

which in view of the lightlike nature of $l_{\alpha}$, satisfies the known relations for null fields

$$
l_{\nu} T^{\nu \mu}=0, \quad T_{\mu}^{\mu}=0, \quad T^{\mu \nu} T_{\mu v}=0 .
$$

An inertial observer characterized by its four-velocity $u^{\alpha}$ will measure a flux of energy or density of four-momentum given by

$$
N^{\alpha}(x)=T^{\alpha \beta} u_{\beta}=\left[R^{2}(x) / 4 \pi\right] l_{\beta} u^{\beta} l^{\alpha} .
$$

As $N^{\alpha}$ is proportional to $l^{\alpha}$, it is also a null vector, whose zero component $N^{\alpha} u_{\alpha}$ gives the energy density as measured by this inertial observer.

\section{APPENDIX: EXISTENCE OF SOLUTIONS}

The algebraic-partial differential system (3.2) is analyzed from the point of view of the differential ideal of Frobenius-Cartan. ${ }^{4}$ Consequently, it will be considered as an exterior differential system, in which $G^{\mu v \sigma \tau}$ is a representation of the metric in the exterior algebra

$$
\begin{aligned}
& \stackrel{0}{\omega} \equiv F_{[\alpha \beta \gamma \delta]}=0 \text {, } \\
& \stackrel{\circ}{\alpha} \equiv * F_{[\alpha \beta \gamma]}-4 \pi * j_{[\alpha \beta \gamma]}, \\
& \stackrel{0}{\omega_{\alpha \beta \gamma \delta}} \equiv P_{\alpha \beta \gamma \delta}-G^{\mu \nu \sigma \tau} P_{\alpha \beta \mu \nu} P_{\sigma \tau \gamma \delta}=0 \text {, } \\
& \stackrel{\circ}{\beta} \equiv G^{\alpha \beta \gamma \delta} G^{\mu v \rho \sigma} \epsilon^{\lambda \pi \tau 0} P_{\lambda \pi \alpha \beta} P_{\tau 0 \mu \nu} F_{\gamma \delta} F_{\rho \sigma}=0 \text {, }
\end{aligned}
$$

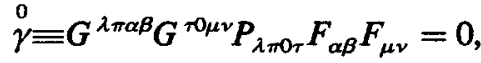

$$
\begin{aligned}
& \stackrel{0}{\omega}_{\lambda \pi v} \equiv G^{\tau 0 \alpha \beta} P_{[\lambda \pi \tau 0 v]} F_{\alpha \beta}+P_{[\lambda \pi \tau 0} G_{, \nu]}{ }^{0 \alpha \beta} F_{\alpha \beta} \\
& +P_{[\lambda \pi 0 \tau} G^{0 \tau \alpha \beta} F_{\alpha \beta v]}=0, \\
& \stackrel{1}{\omega}_{\lambda \pi} \equiv d F_{\lambda \pi}-F_{\lambda \pi v} d x^{v}=0 \text {, } \\
& \stackrel{1}{\omega}_{\lambda \pi \tau 0} \equiv d P_{\lambda \pi \tau 0}-P_{\lambda \pi \tau 0 v} d x^{v},
\end{aligned}
$$


plus the closure, i.e., the exterior differential of the system (A1), that we symbolically write down

$$
d(\mathrm{~A} 1) \text {. }
$$

Equations (A1) and (A2) are defined on a 139-dimensional manifold $N=N\left(\kappa^{\alpha}, F_{\mu \nu}, P_{\alpha \delta \sigma \tau}, F_{\alpha \beta \gamma}, P_{\gamma \delta \mu v \tau}\right)$, because the electromagnetic field source and the geometry of the base space (space-time) are considered to be given.

After a lengthy but easy calculation it is proven that we recover system (3.2) when the complete exterior system (A1) and (A2) is restricted to the original manifold, and its integrability conditions are automatically satisfied!

Therefore, the equations we propose to define the radiative electromagnetic field as the contraction $P_{\alpha \beta \gamma \delta} F^{\gamma \delta}$ have solutions. It remains to prove mathematically its uniqueness.
'E. Konopinski, Electromagnetic Fields and Relativistic Particles (McGraw-Hill, New York 1981).

${ }^{2}$ F. Rohrlich, Classical Charged Particles (Addison-Wesley, Reading, MA, 1965).

${ }^{3}$ V. Ditkine and A. Provdnikov, Transformations Intégrals et Calcul Opérationnel (Mir, Moscow, 1978).

${ }^{4} Y$. Choquet-Bruhat and C. De Witt-Morette, with M. Dillar-Bleick, Analysis, Manifolds and Physics (North-Holland, Amsterdam, 1982), revised edition.

${ }^{5}$ G. F. R. Ellis, "Relativistic Cosmology," in Proceedings of the International School of Physics "Enrico Fermi," Course XLVII, edited by B. K. Sachs (North-Holland, Amsterdam, 1971).

${ }^{6}$ A.Sommerfeld, Electnodynamics (Academic, New York, 1952); Partial Differential Equations in Physics (Academic, New York, 1967).

'V. P. Frolov, "The Newman-Penrose method in the theory of General Relativity," in Proceeding (Trudy) of the P. N. Lebedev Physics Institute, Vol. 96, edited by N. G. Basov (Consultants Bureau, New York, 1979).

${ }^{8}$ V. L. Ginzburg, Sov. Phys. Usp. 98, 569 (1969).

${ }^{9} \mathrm{M}$. Walker, in Isolated Gravitating Systems in General Relativity, edited by J. Ehlers (North-Holland, Amsterdam, 1979). 